\title{
Influence of 3D Printed Porous Architecture on Mesenchymal Stem Cell Enrichment and Differentiation
}

Kimberly M. Ferlin ${ }^{1}$, Margaret E. Prendergast ${ }^{1}$, Makenzie L. Miller ${ }^{1}$, David S. Kaplan ${ }^{2}$, and John P. Fisher ${ }^{1} *$

${ }^{1}$ Fischell Department of Bioengineering, University of Maryland, College Park, Maryland, ${ }^{2}$ U.S. Food and Drug Administration, Center for Devices and Radiological Health, Silver Spring, Maryland

Short Title:

Key Words:

Submitted To:

*Corresponding Author:
3D Printed Scaffolds for MSC Enrichment and Differentiation

3D printing, biomaterial, mesenchymal stem cell, differentiation, scaffold architecture

Acta Biomaterialia

John P. Fisher, Ph.D.

Fischell Family Distinguished Professor and Associate Chair

Director of Graduate Studies

Fischell Department of Bioengineering

University of Maryland

3238 Jeong H. Kim Engineering Building

College Park, Maryland 20742

Work Phone: 3014057475

Work Fax: 3014050523

Email: $\quad$ jpfisher@umd.edu

Web: http://www.glue.umd.edu/ jpfisher

The items identified by brand name are the choice of the authors' and are not to be construed as conveying an official endorsement by the U.S. Food and Drug Administration. 


\section{Abstract}

2 The interactions between cells and an underlying biomaterial are important for the promotion of

3 cell adhesion, proliferation, and function. Mesenchymal stem cells (MSCs) have great clinical

4 potential as they are an adult stem cell population capable of multilineage differentiation. The

5 relationship between MSC behavior and several material properties including substrate stiffness

6 and pore size are well investigated, but there has been little research on the influence of porous

7 architecture in a three-dimensional scaffold with a well-controlled architecture. Here, we

8 investigate the impact two different three-dimensionally printed, pore geometries on the

9 enrichment and differentiation of MSCs. 3D printed scaffolds with ordered cubic pore geometry

10 were supportive of MSC enrichment from unprocessed bone marrow, resulting in cell surface

11 marker expression that was comparable to typical adhesion to tissue culture polystyrene, the gold

12 standard for MSC culture. Results also show that scaffolds fabricated with ordered cubic pores

13 significantly increase the gene expression of MSCs undergoing adipogenesis and

14 chondrogenesis, when compared to scaffolds with ordered cylindrical pores. However, at the

15 protein expression level, these differences were modest. For MSCs undergoing osteogenesis,

16 gene expression results suggest that cylindrical pores may initially increase early osteogenic

17 marker expression, while protein level expression at later timepoints is increased for scaffolds

18 with ordered cubic pores. Taken together, these results suggest that $3 \mathrm{D}$ printed scaffolds with

19 ordered cubic pores could be a suitable culture system for single-step MSC enrichment and

20 differentiation. 


\section{Introduction}

23 Mesenchymal stem cells (MSCs) are an adult stem cell population found in multiple tissues

24 throughout the body including bone marrow, adipose tissue, the synovial membrane, and

25 trabecular bone. MSCs are of particular interest for therapeutic applications because the cells

26 can be differentiated into a number of lineages including chondrocytes, adipocytes, and osteoblasts [1]. MSCs exist in very low numbers in the highly cellular and heterogeneous bone marrow, and lack unique identifying markers necessary for definitive isolation. Isolation and characterization of MSCs is currently based on a set of established properties set forth by the International Society for Cellular Therapy [2]. Based on these properties, MSCs must show: adherence to tissue culture polystyrene (TCPS), the expression of a panel of positive cell surface markers, the lack of expression for a panel of negative surface markers, and the ability to undergo trilineage differentiation into osteoblasts, adipocytes, and chondrocytes [2,3]. Standard protocols for the isolation of MSCs from the bone marrow include extraction of whole bone marrow, density gradient centrifugation, and expansion of the mononuclear cell fraction on TCPS. While this protocol is well accepted for the use of MSCs, there is evidence to suggest that this expansion phase may result in phenotypic changes in MSCs [4, 5], including a loss of differentiation potential and cell senescence. The use of biomaterials for the enrichment and expansion of MSCs in a more physiologically relevant three dimensional (3D) environment is of growing interest in the field, with several successful attempts to expand MSCs in 3D with the use of microcarriers under dynamic conditions [6-8]. However, these strategies do not eliminate the need for initial culture on plastic.

Although the use of MSCs as a therapeutic agent is promising, there is still much to learn about the native MSC niche and how these properties can be exploited for regenerative medicine applications. The MSC niche is a complex and dynamic structure consisting of cellular components, secreted factors, extracellular matrix, and physical factors among others [9]. The influence of physical parameters such as substrate elasticity and geometry on MSCs is of rising interest in the field of tissue engineering, especially with the development of novel micropatterning techniques [10]. With the rise of new technologies, substrates can be controlled

51 such that studies can be completed on the single cell level. Substrate stiffness has been shown to 52 play a large role in stem cell differentiation, with softer substrates resulting in neurogenic or 
53 adipogenic differentiation while more rigid substrates are favorable for osteogenic differentiation

54 [11]. The investigation of substrate shape and its relationship to cell contraction and

55 differentiation has been well characterized [10, 12, 13] using micropatterning techniques. These

56 studies have shown that culturing cells in geometries that permit cell spreading and therefore

57 higher levels of contractility promote osteogenic phenotypes, even when cells are exposed to a

mixture of chemical cues. Likewise, under an identical chemical environment, rounded geometries encouraged low cell tension and an adipogenic phenotype [10].

The discovery of the link between cell shape and differentiation has been critical in the design of biomaterials that can be used for cell therapy applications. However, many of these fundamental studies have been conducted in two dimensional (2D) or "pseudo"- 3D [12] substrates, which is dissimilar to the native environment where cells may be implanted for regenerative therapy. Here, we aim to elucidate the relationship between 3D geometry and MSC enrichment and differentiation through the use of 3D printed biodegradable scaffolds. 3D printing technology provides a means to fabricate highly ordered and complex scaffold structures with great reproducibility. The design criteria for scaffolds capable of supporting MSC enrichment and differentiation includes a structure that consists of an interconnected porous network with pore sizes on the range of hundreds of microns [14-16], mechanical properties, such as modulus (0.03-1600 MPa), in the range of mesenchymal tissues, and mechanical stability during in vivo

72 degradation [17]. Previous studies, from our laboratory and others, have investigated the impact

73 of various aspects of 3D printed scaffolds on MSC behavior, including substrate stiffness, pore

74 size, and substrate composition [18-20]. This has been especially advantageous for applications

75 involving interfacial tissue engineering, where 3D printing can be used to recapitulate the

76 complex architecture of the tissue interface [21, 22].

Here, our first objective was to study the impact of 3D pore structure using two pore

79 architectures printed with the synthetic polymer poly(propylene fumarate) (PPF). PPF is a

80 biodegradable polymer that can form crosslinked networks through its carbon-carbon double

81 bond, and has been used extensively in tissue engineering applications [19, 23-25]. Scaffolds

82 were designed to have an interconnected porous network with pores that exhibited cubic or 83 cylindrical geometry. 
85 Using 3D printed PPF scaffolds, we hypothesized that MSC enrichment in 3D can equal or surpass that of traditional TCPS due to the ability of the scaffold to encourage 3D interactions native to the cell population. Further, we hypothesized that the response of MSCs to chemical cues known to induce differentiation will be influenced by the pore architecture of the 3D printed scaffold.

\section{Materials and Methods}

\subsection{Scaffold Design}

93 Three-dimensional scaffolds composed of cubic or cylindrical pore geometries were designed

94 using SolidWorks ${ }^{\circledR}$ (Waltham, MA). All scaffolds were designed to have an ordered pattern of

95 continuous pores, resulting in an interconnected porous network. Scaffolds were constructed to

96 have a theoretical porosity of $80 \%$ and a pore volume of $1 \mathrm{~mm}^{3}$. Several iterations of the each

97 design were tested until an interconnected porous network and high reproducibility were

98 achieved.

\subsection{Poly(Propylene Fumarate) Synthesis and 3D Printing}

101 All scaffolds were fabricated out of a polymer resin consisting primarily of PPF. PPF was

102 synthesized using a previously established protocol [26], and the number average molecular

103 weight $\left(\mathrm{M}_{\mathrm{n}}\right)$ and polydispersity index (PDI) were determined by gel permeation chromatography.

104 The $\mathrm{M}_{\mathrm{n}}$ of PPF used to synthesize all scaffolds in this study was 1133-1987 Da with a PDI of

105 1.8-2.1. Scaffolds were fabricated using an EnvisionTEC Perfactory ${ }^{\circledR} \mathrm{P} 4$ with an exposure to

$106 \mathrm{UV}$ light of $350 \mathrm{~mW} / \mathrm{dm}^{2}$ for 100 s per layer. Scaffolds were printed out of polymer resin that

107 was composed of PPF and its monomer diethyl fumarate (DEF) in a 1:0.8 ratio as well as the

108 photoinitiator bis (2,4,6-trimethylbenzoyl) henylphosphine oxide (BAPO, 1\%), and

109 photoinhibiting dyes HMB (1\%) and $\alpha$-tocopherol $(0.1 \%)$. Following printing, several post-

110 processing methods were investigated to achieve the desired scaffold properties, including

111 optimization of solvent, length of exposure to solvent, and post-curing. Following optimization,

112 it was determined that the most effective post-processing method was for scaffolds to be

113 immediately placed in a 30 min wash of isopropyl alcohol, followed by a 30 min wash of $100 \%$

114 acetone to remove any uncured resin from within the porous network. After these washes, 
115 scaffolds were post-cured in a 3D systems UV box at a total energy of 110J. Following post-

116 curing, scaffolds were rinsed overnight in a solution of $70 \%$ ethanol to remove any remaining

117 unreacted resin or debris. For characterization studies, scaffolds were vacuum dried overnight

118 prior to testing. For cellular studies, scaffolds were sterilized in $70 \%$ ethanol for 4 hours

119 followed by 4, 30min washes in sterile phosphate-buffered saline (PBS) to remove any traces of

120 ethanol.

121

\subsection{Soluble Fraction Determination}

123 To evaluate PPF crosslinking and the effectiveness of the post processing methods, the cured 124 network soluble fraction, or sol fraction was determined using previously established methods

125 [23]. The sol fraction is the percent of soluble polymer remaining after post processing.

126 Scaffolds were weighed $\left(\mathrm{W}_{\mathrm{i}}\right)$ prior to immersion in acetone, which served as the extraction

127 solvent. The samples were incubated in acetone for 24 hours. Following incubation, samples

128 were completely dried and weighed again $\left(\mathrm{W}_{d}\right)(\mathrm{n}=5)$. The sol fraction was calculated using the 129 equation:

$$
\text { sol fraction }=\frac{W_{i}-W_{d}}{W_{i}} \times 100 \%
$$

\subsection{Compressive Mechanical Testing}

132 Compressive mechanical testing of scaffolds was conducted using a mechanical testing system

133 (Model 858, MTS System Corporation, Eden Prairie, MN). Force and displacement were zeroed

134 prior to compression, with the top plate slightly above the surface of the sample. Samples $(\mathrm{n}=5)$

135 were compressed at a crosshead speed of $2 \mathrm{~mm} / \mathrm{min}$ while force and displacement were

136 monitored throughout the experiment and converted to stress and strain following completion.

137 The experiment was halted after sample fracture. The elastic modulus for each sample was

138 calculated using MATLAB to determine the slope of the linear region of the stress-strain curve

139 as previously described [27]. Briefly, the linear region was calculated by using the linear fit

140 command starting with the first 10 data points, considering a preload of $1 \mathrm{~N}$. The program

141 continued to systematically add points in steps of 8 until the $\mathrm{R}^{2}$ value dropped below 0.95 . The

142 slope of this region is representative of the elastic modulus of the sample. 


\subsection{Micro-Computed Tomography}

Micro-computed tomography $(\mu \mathrm{CT})$ was used as a means to noninvasively image and characterize scaffold parameters. Scanning was performed using a $\mu$ CT 100 (SCANCO Medical, Brüttisellen, Switzerland) operated at $70 \mathrm{kVp}, 9 \mathrm{~mm}$ isotropic voxels, and $200 \mathrm{~mA}$. The 3D datasets that resulted from each scan were segmented using predetermined thresholds (lower: 35, upper: 188, maximum: 1000), with gauss sigma (0.8) and support (1) values to distinguish pores from polymer [18]. All resulting 3D images were evaluated using the Image Processing Language (IPL) from Scanco to determine pore size and porosity. Three samples of each scaffold type were scanned.

\subsection{Unprocessed Human Bone Marrow Culture}

Whole unprocessed human bone marrow (Lonza, Walkersville, MD) was seeded onto cubic pore scaffolds that were either unmodified or modified to contain type I collagen (Corning, Corning, NY), a mixture of type I collagen and hyaluronic acid (HA, Sigma), or TGF- $\beta 3$ (R\&D Systems, Minneapolis, MN) on the surface of the scaffold. The surface of PPF scaffolds were modified using EDC/NHS chemistry. Briefly, scaffolds were immersed in 50mM MES buffer $(\mathrm{pH}=5.5$, Sigma) containing 0.5M N-(3-dimethylaminopropyl)-N'-ethylcarbodiimide hydrochloride (EDC, Sigma) and $0.5 \mathrm{M} \mathrm{N}$-hydroxysuccinimide (NHS, Sigma) for 14 hours at room temperature, followed by a wash in PBS to remove any excess byproducts. For protein modification, scaffolds were modified with type I collagen or a mixture of type I collagen and HA at $1 \mathrm{mg} / \mathrm{mL}$. For growth factor modification, scaffolds were modified with a solution of $10 \mu \mathrm{g} / \mathrm{mL}$ of TGF- $\beta 3$. All solutions were made up in PBS and sterile filtered prior to incubation with the EDC/NHS activated scaffolds for 1 hour at room temperature. Following modification, scaffolds were rinsed 3 times in sterile PBS to remove any unbound protein or growth factor. Tissue culture plastic (TCPS) served as a control. Groups were incubated at $37^{\circ} \mathrm{C}, 5 \% \mathrm{CO} 2$, and $80 \%$ humidity until experimental analysis. Media (growth media described below) was changed every 2 days.

\subsection{Evaluation of Mesenchymal Stem Cells Isolated from Whole Bone Marrow}

To analyze the cell population that was captured from unprocessed bone marrow, cells were subjected to flow cytometry analysis using a Human MSC (hMSC) Analysis Kit (BD Stemflow, BD, Franklin Lakes, NJ). At days 1 and 7, cells were lifted from the surface of the scaffold or 
175 TCPS and resuspended at a concentration of $5 \times 10^{6}$ cells $/ \mathrm{mL}$. Cells were stained following the 176 manufacturer's protocol. Briefly, $100 \mu \mathrm{L}$ of each cell suspension was mixed with $20 \mu \mathrm{L}$ of the

177 hMSC Positive Cocktail (CD90 FITC, CD105 PerCP-Cy5.5, and CD 73 APC) and $20 \mu \mathrm{L}$ of the 178 hMSC Negative Cocktail (CD34 PE, CD11b PE, CD19 PE, CD45 PE, and HLA-DR PE) and 179 incubated at $4^{\circ} \mathrm{C}$ in the dark for 30 minutes. Following incubation, the cell suspensions were 180 washed $2 \mathrm{x}$ with $500 \mu \mathrm{L}$ of PBS containing $1 \%$ BSA. After washing, cells were resuspended in $181500 \mu \mathrm{L}$ of PBS containing 1\% BSA and analyzed using a BD FACSCanto II to quantify the 182 amount of each marker present. Human MSCs at P4 (Lonza) were used as a control, and to set 183 up an analysis template for all samples.

\subsection{Human Mesenchymal Stem Cell Culture and Scaffold Seeding}

186 Human MSCs (Lonza) were expanded prior to the study on tissue culture polystyrene flasks in 187 media consisting of DMEM (Life Technologies, Frederick, MD) supplemented with 10\% fetal 188 bovine serum (Life Technologies), 1.0\% v/v penicillin/streptomycin (Life Technologies), $0.1 \mathrm{mM}$ non-essential amino acids (Life Technologies), and 4mM L-glutamine (Life Technologies)

190 following manufacturer's protocols and previously described methods [28]. Media was changed 191 every 3 days according to the manufacturer's specifications, and cell cultures were incubated at $19237^{\circ} \mathrm{C}, 5 \% \mathrm{CO} 2$, and $80 \%$ humidity. Cells at passage 4 were used for all experiments. Prior to 193 cell seeding, adherent MSCs were lifted with trypsin/EDTA (Life Technologies) and counted 194 using trypan blue uptake to determine viability. MSCs were seeded onto sterilized scaffolds in a 19548 well plate. Each scaffold was seeded with approximately 42,000 cells $\left(11,000 \mathrm{cells} / \mathrm{cm}^{2}\right)$ in a concentrated solution of $20 \mu \mathrm{L}$ and allowed to adhere for 1 hour at $37^{\circ} \mathrm{C}$ before additional media

197 was added.

\subsection{XTT Assay}

200 To quantitatively evaluate cell metabolic activity on the scaffolds surface, a Cell Proliferation Kit 201 II (XTT) (Roche, Mainheim, Germany) was used. XTT [2,3-bis-(2- methoxy-4-nitro-5202 sulfophenyl)-2H-tetrazolium-5-carboxanilide] was used following the manufacturer's protocols. 203 The electron coupling and XTT labeling reagents were thawed and immediately combined in a 204 1:50 ratio. Scaffolds were moved into new cell culture wells to avoid evaluating cells that did 205 not attach to the scaffold surface. The XTT solution was added to cell culture wells and 
206 incubated at $37^{\circ} \mathrm{C}$ for 4 hours. Following incubation, absorbance was measured with an M5

207 SpectraMax plate reader (Molecular Devices, Sunnyvale, CA). Net absorbance was calculated

208 (A450-A650) for each sample and reported. Cells grown on tissue culture polystyrene at the

209 same density served as a control. Samples were evaluated at day 1, 4, and 7.

2112.10 Fluorescence Imaging

212 The viability of cells seeded onto scaffolds was qualitatively evaluated using live/dead

213 fluorescent staining. At days 1, 4, and 7, scaffolds were soaked in PBS containing $1 \mathrm{mM} \mathrm{CaCl} 2$

214 and $0.5 \mathrm{mM} \mathrm{MgCl}_{2}$ for $30 \mathrm{~min}$ to remove non-adherent cells as well as any remaining medium.

215 Following standard protocols, scaffolds were incubated with $2 \mathrm{mM}$ ethidium homodimer and

$2164 \mathrm{mM}$ calcein AM (Life Technologies) for 30min. Images were taken using a fluorescent

217 microscope (Axiovert 40 CFL with filter set 23; Zeiss, Thornwood, NY) equipped with a digital

218 camera (Diagnostic Instruments 11.2 Color Mosaic, Sterling Heights, MI).

\subsection{Induction of MSC Differentiation}

221 To evaluate the impact of pore geometry on human MSC differentiation, MSCs were seeded

222 onto each scaffold type and induced down the three main mesenchymal lineages (osteogenesis,

223 chondrogenesis, and adipogenesis). Cells were seeded onto scaffolds as described above.

224 Following incubation for 24 hours in growth medium, cells were cultured for 21 days in

225 osteogenic (DMEM with 10\% FBS, 1mM sodium pyruvate (Life Technologies), 100U/100 $\mu \mathrm{g}$

226 penicillin-streptomycin, $10^{-7} \mathrm{M}$ dexamethasone (Sigma-Aldrich), 50 $\mu \mathrm{g} / \mathrm{mL}$ ascorbic acid (Sigma-

227 Aldrich), and 10mM $\beta$-glycerophosphate), chondrogenic (high glucose DMEM supplemented

228 with ITS (BD), 4mM L-proline (Sigma-Aldrich), 50 $\mathrm{g} / \mathrm{mL}$ ascorbic acid, $1 \%$ sodium pyruvate,

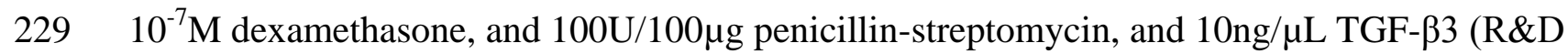

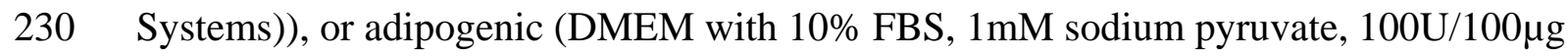

231 penicillin-streptomycin, 10-6M dexamethasone, $10 \mu \mathrm{g} / \mathrm{mL}$ insulin (Sigma-Aldrich), $0.5 \mathrm{mM}$

232 IBMX (Life Technologies), and 200 $\mu$ M indomethacin (Life Technologies)) media as described

233 previously [29]. MSCs cultured in growth media served as a control. All media were changed

234 every 3 days. At days 7, 14, and 21, samples were collected for RNA isolation and histological

235 analysis, by lifting the cells from the surface of the scaffolds. The number of cells remaining on 
the scaffolds was not quantified, though it is not anticipated that a significant cell population remained after exposure to trypsin.

\subsection{Quantitative Reverse Transcriptase-Polymerase Chain Reaction}

240 RNA was isolated using an RNeasy Mini Plus Kit (Qiagen, Valencia, CA) following standard 241 protocols. Isolated RNA was then reverse transcribed to cDNA using a High Capacity cDNA 242 Archive Kit (Applied Biosystems, Foster City, CA). The sequences of the gene expression 243 assays used (Applied Biosystems) are proprietary. Glyceraldehyde-3-phosphate dehydrogenase

244 (GAPDH, Hs99999905_m1) served as an endogenous control gene for all samples. Alkaline 245 phosphatase (ALP, Hs00758162_m1) and osteopontin (OPN, Hs00960641_m1) were assayed as 246 markers of early and late osteogenesis, respectively. Adiponectin (Hs00605917_m1) and type II 247 collagen (Hs00264051_m1) were assayed as markers for adipogenesis and chondrogenesis, 248 respectively. Gene expression assays were combined with the cDNA to be analyzed and 249 TaqMan PCR Master Mix (Applied Biosystems). The reaction was performed on a 7900HT 250 real-time PCR System (Applied Biosystems) using thermal conditions of 2 minutes at $50^{\circ} \mathrm{C}, 10$ 251 minutes at $95^{\circ} \mathrm{C}$, and 40 cycles of 15 seconds at $95^{\circ} \mathrm{C}$ and 1 minute at $60^{\circ} \mathrm{C}$. The relative gene 252 expression level of each target gene was then normalized to the mean of the GAPDH in each 253 group. Fold change was calculated using the $\Delta \Delta \mathrm{CT}$ relative comparative method as described 254 previously [28]. Samples were completed in triplicate and standard deviations are reported $(\mathrm{n}=$ $2553)$.

\subsection{Histological Analysis}

258 At days 7, 14, and 21, cells were lifted from the surface of the scaffolds and seeded onto 96 well

259 plates for 24 hours. At this time cells were fixed with $10 \%$ formalin for 10 minutes, and stored

260 at $4^{\circ} \mathrm{C}$ in PBS. Histological staining was completed to show calcification, glycosaminoglycan 261 production, and the presence of lipid droplets. Mineralization was visualized using alizarin red 262 (Sigma) staining following standard protocols. Briefly, freshly prepared 2\% w/v alizarin red 263 solution was added to cell monolayers for 5 minutes. Cells were washed 3 times in DI water and 264 imaged immediately. Glycosaminoglycan production was visualized by staining with $0.5 \%$ 265 Alcian Blue solution (Poly Scientific, Bay Shore, NY) followed by a nucleic counter stain with 266 Nuclear Fast Red (Poly Scientific). For the visualization of lipid vacuoles in adipogenic 
samples, a working solution of Oil Red O (Poly Scientific) was prepared and applied to fixed monolayers, followed by counterstaining with hematoxylin. For quantification, 5 x 5 tiled images (total tiled image area $=3.177 \mathrm{~mm}^{2}$ ) were captured of each well $(\mathrm{n}=4)$ using an inverted

270 microscope (Zeiss AxioVert, equipped with an AxioCam MRm digital camera; Zeiss, Inc.,

271 Thornwood, NY) and AxioVision Software (Version 4.8.2; Zeiss, Inc.). A routine was created in

272 Axiovision for each stain to capture and calculate regions positively stained for calcification,

273 lipid deposits, or proteoglycans.

\subsection{Statistical Analysis}

276 All data was analyzed using one-way analysis of variance and Tukey's multiple-comparison test

277 to determine statistical differences between scaffolds. A confidence interval of $95 \%(\alpha=0.05)$ was used for all analyses, and means and standard deviations are shown in each figure.

\section{Results}

281 Scaffolds were designed to have an interconnected cubic or cylindrical porous network with

$2821000 \mu \mathrm{m}$ pore size and $80 \%$ porosity. In order to achieve an interconnected porous network that

283 could be reproducibly printed, several design changes were made including altering the design of

284 the pores within the scaffold backbone, the pore side length or diameter, and the wall thickness

285 between pores. Using $\mu \mathrm{CT}$, we were able to evaluate the actual scaffold parameters that resulted

286 following fabrication and post-processing (Figure 1). The optimized printed scaffolds showed

287 good correlation to the designed parameters, with cubic pore and cylindrical pore scaffolds

288 having an actual porosity of $78.8 \pm 1.8 \%$ and $70.4 \pm 11.8 \%$ respectively. Pore size also printed

289 close to the theoretical values with cubic pore scaffolds resulting in a pore size of $882.0 \pm 68.0$

$290 \mu \mathrm{m}$ and cylindrical pore scaffolds a pore size of $735.0 \pm 91.0 \mu \mathrm{m}$. By using $\mu \mathrm{CT}$ to generate a

291 pore map of each scaffold, it was apparent that the pores regardless of architecture were

292 continuous and interconnected throughout the scaffold.

294 Imaging with $\mu \mathrm{CT}$ also showed that the scaffold integrity was not compromised by the use of 295 chemical washes in post processing, as there are few areas of the scaffold surface that are 296 roughened by surface defects. Similarly, there is little to no remaining debris around the scaffold 297 surface. Sol fraction testing revealed that all methods tested had a sol fraction of less than 5\%. 
298 The post processing methods used were effective at removing any remaining uncured polymer

299 resin which could be potentially damaging to cell viability (Figure 3A).

Compressive mechanical testing (Figure 2A) revealed that there was an increase in the elastic modulus of the scaffolds with an increasing amount of total energy delivered to the scaffold using post printing UV crosslinking. For both acetone wash conditions, $110 \mathrm{~J}$ resulted in significantly lower modulus, followed by $550 \mathrm{~J}$. There were no statistical differences between 1100 and $2200 \mathrm{~J}$ for either acetone wash. When comparing batches of PPF, it was found that there is a correlation between the molecular weight of the polymer and the elastic modulus, with higher molecular weights resulting in increased moduli of the printed scaffolds. Comparing geometries, cubic pore scaffolds had a statistically higher elastic modulus of 25.9 MPa, when compared to 17.5 MPa for cylindrical pore scaffolds (Figure 2B). However, this difference, though statistically significant, is modest compared to those thought to influence stem cell fate

Flow cytometry analysis for the panel of positive surface markers for human MSCs was

314 completed on days 1 and 7 following bone marrow seeding. Results show that as early as day 1,

315 scaffolds modified with biomolecules and unmodified scaffolds demonstrate expression levels

316 equivalent to the TCPS control (see Table 1). Scaffolds with surface modification have a 317 marginally increased expression of positive surface markers. Scaffolds modified with a mixture 318 of collagen and HA, as well as scaffolds modified to contain the growth factor TGF- $\beta 3$, showed 319 the highest level of stem cell marker expression at day 1, with levels above that of the TCPS 320 control. On day 7, all experimental groups again show stem cell marker expression levels 321 similar to the control. Here, both the unmodified scaffold group and the collagen modified 322 scaffold group exhibit stem cell marker expression levels that are increased over the control, with 323 the collagen modified scaffold group having the highest overall expression compared to all 324 groups.

326 The results of the XTT assay show that MSCs cultured on scaffolds over a period of 7 days 327 exhibit levels of metabolic activity that are not statistically different from cells cultured on tissue culture polystyrene (Figure 3A). Quantitative results were confirmed using qualitative imaging 
techniques. In Figure 3B, the majority of the cells stain positively for calcein AM (green) indicating that they are viable on the scaffold surface and within the pores of the scaffold. An increased level of fluorescence within the pores suggests that MSCs have begun to fill the pores.

332 Taken together, these results confirm that the post processing methods used to remove unreacted

333 polymer following fabrication is effective and promotes cell function on and within the scaffold

334 architecture. We did not distinguish between cells cultured on the interior or exterior of the

335 scaffold, but cells on the scaffold as a whole. As the pore sizes of our scaffolds are on the range

336 of hundreds of microns, we do not anticipate that there should be oxygen or diffusional

337 differences between the interior and exterior of the scaffold.

Human MSC differentiation was assessed over 21 days using qRT-PCR and histological staining for markers of each mesenchymal tissue. Gene expression analysis shows that cylindrical pore scaffolds appear to promote an early increase in osteogenic markers (see Figure 4A and B)

342 following exposure of the cells to osteogenic media. Both ALP and OPN show a statistically 343 greater expression of the respective marker in the cylindrical pore group at day 7 when compared

344 to the cubic pore group within the same timepoint. This initial difference is diminished at later

345 timepoints, with the results of histological analysis indicating that the cubic pore group appears

346 to catch up and pass the cylindrical pore group in terms of calcification, as indicated through the

347 quantification of alizarin red histological staining for calcified regions. Image analysis results

348 show that at day 21 , the cubic pore group shows a significantly greater number of calcified

349 regions when compared to the cylindrical pore group (Figure 5).

For adipogenesis, the cubic pore group at day 21 shows significantly increased expression of

352 adiponectin, when compared to the corresponding cylindrical pore group (see Figure 4C). For 353 both pore geometries, the expression of adiponectin is significantly upregulated on day 21.

354 Based on histological evidence, there appears to be no significant difference between pore

355 geometries when quantifying the number of lipid deposits per cell for both groups.

357 As with the adipogenic group, the cubic pore samples showed significantly upregulated type II 358 collagen expression compared to the cylindrical pore samples for the group undergoing chondrogenesis (see Figure 4D). At day 21, both cubic and cylindrical pore scaffolds show an 
360 upregulation of type II collagen expression, with cubic pore scaffolds resulting in the highest

361 expression. Based on histological evidence, there appears to be no significant difference

362 between pore geometries when quantifying the number of proteoglycan-rich regions per cell for

363 both groups.

\section{Discussion}

366 The ability to both enrich MSCs from isolated tissue and subsequently control MSC

367 differentiation is of great importance to the field of cell therapy and regenerative medicine. In

368 addition to chemical and biomolecular cues, MSCs have been shown to respond to scaffold

369 parameters including substrate stiffness, surface roughness, pore size, and 2D geometry, such as

370 curvature. In the work presented here, we used 3D printing technology to evaluate the impact of

$3713 \mathrm{D}$ pore geometry on human MSC enrichment and differentiation. Scaffolds were printed out of

372 a resin of PPF, a biodegradable polymer that has previously been shown to result in accurately

373 printed scaffolds, with parameters such as pore size, porosity, and wall thickness exhibiting

374 accuracies of up to $91 \%$ [18]. One of the most important criteria for the culture and

375 differentiation of MSCs on 3D scaffolds is an interconnected porous network to promote the

376 exchange of nutrients, soluble factors, and cell signals. Here, we showed the ability to achieve

377 an interconnected network of evenly sized pores that could be printed with high reproducibility.

378 Scaffolds were designed to be composed of repeatable units such that feature size and shape

379 could be easily altered. Design parameters were altered based on the outcome of each print.

380 PPF is a viscous polymer, a characteristic that can be troublesome for printing systems based on

381 the use of liquid resins. Polymer resin viscosity has been noted in previous studies as a potential

382 source of printing inaccuracies, particularly for small feature sizes [30]. Lowering of the

383 viscosity through the use of a solvent is a simple way to combat this issue, as we have done here

384 using DEF, the monomer of PPF.

386 PPF forms a crosslinked network through the crosslinking of a carbon-carbon double bond 387 within the backbone of the PPF chain. DEF also contains a crosslinkable carbon-carbon double 388 bond and therefore serves as an excellent candidate for the dilution of PPF to achieve a desirable 389 viscosity for printing. Previous studies have shown that the inclusion of a percentage of DEF

390 within the PPF resin improves the mechanical properties of the scaffold, as it increases the 
number of crosslinks formed between polymer chains [23]. PPF is a hydrolytically degradable polymer, with fumaric acid and propylene glycol as the byproducts of degradation. While the degradation of the scaffolds used in the current study was not investigated, our lab has previously studied how 3D printed PPF scaffolds degrade in vitro. During a 224 day degradation period, the structural, mechanical, and cytocompatibility properties of three different 3D printed PPF scaffold designs were evaluated [17]. Results indicate that the scaffolds undergo surface degradation, which was confirmed through an increase in $\mathrm{pH}$ and the formation of micropores and surface channels. Additionally, extracts of the degradation products were found to not induce significant cell death.

PPF scaffolds with cubic pores were first investigated for their utility in MSC enrichment from unprocessed human bone marrow. Here, we showed that cubic pore scaffolds, both unmodified 403 and modified with biomolecules, supported the attachment of cells with similar phenotypic 404 signatures to those cultured on TCPS, the current gold standard for MSC enrichment and expansion. Through the direct seeding of whole bone marrow onto 3D scaffolds, naïve and early progenitor cells are preserved, which are hypothesized to be lost during density gradient centrifugation $[5,31]$. In addition to preserving these cell types, direct seeding into 3D scaffolds results in cells maintaining their native morphology, which has been shown to influence MSC proliferation and differentiation capacity [32]. Through the use of surface modification techniques, we were able to attach proteins from the MSC ECM as well as growth factors to the material surface. For MSC isolation and enrichment, there were not large differences between

412 modified and unmodified scaffolds, though there were modest increases in the expression of 413 surface markers for those modified with type I collagen and TGF- $\beta 3$ on day 7 compared to 414 unmodified scaffolds and TCPS. While these increases may indicate that surface modification 415 improves the scaffold for MSC enrichment, further studies need to be completed to elucidate the 416 role of biochemical cues in addition to physical cues for the 3D enrichment of MSCs. In 417 addition to playing a role in enrichment, surface modifications may be of greater importance for 418 the priming of cultures for differentiation towards a specific mesenchymal lineage.

420 In order to elucidate how MSCs would respond to cubic and cylindrical pore geometries 421 presented, cell viability had to be first tested to ensure that the post-processing methods did 
422 negatively affect cell viability. Culture over a 7 day period showed that cells had a metabolic

423 activity level that was not significantly different from the control, indicating that the post-

424 processing methods used resulted in scaffolds that supported MSC cell viability. Differentiation

425 of MSCs down the three main mesenchymal lineages was assessed over a period of 21 days and

426 pore geometries were compared for each group at each timepoint. Results indicated that there

427 were significant differences between the gene expressions of phenotypic markers depending on

428 the 3D pore geometry of the scaffold.

430 The pore sizes of our scaffolds are around $830 \mu \mathrm{m}$ for the cubic pore design and $730 \mu \mathrm{m}$ for the 431 cylindrical pore design. There have been numerous studies that have investigated the influence 432 of pore size for mesenchymal target tissues, especially bone and cartilage. Here we aimed to 433 minimize the impact of pore size by designing the pores to be equivalent in size, and focus on the 434 impact of pore geometry on cell behavior, a concept that has been much less studied. Trabecular 435 bone has a macroporous structure, with open marrow space pores on the order of $1 \mathrm{~mm}$ in size 436 [33]. Previous studies have shown that larger pore sizes (on the order of 1-3 mm) allow for 437 greater interaction between cells and the underlying biomaterial, improving the osteogenic 438 properties of the tissue and resulting in a thicker tissue formation along the material surface [34,

439 35]. For chondrogenesis applications, there are a large range of pore sizes that have been 440 investigated, with disagreement over which size is appropriate for the promotion of the 441 chondrogenic phenotype. Studies have indicated that smaller pore sizes (on the range of 150 $442 \mu \mathrm{m})$ are successful for the promotion of type II collagen and aggrecan production [14], while 443 larger pores $(250-500 \mu \mathrm{m})$ increased cell proliferation and ECM deposition [15]. Additional 444 studies investigating scaffold permeability showed that pore sizes on the order of $900 \mu \mathrm{m}$ 445 supported cartilage matrix production both in vitro and in vivo [36, 37]. While the feature sizes 446 in this study are greater than the subcellular curvatures that are commonly investigated in 2D 447 studies of cell behavior [10, 13], we showed that scaffolds fabricated with larger pore sizes were 448 capable of supporting MSC attachment, proliferation, and differentiation. Due to the tunable 449 nature of our scaffold designs, the future investigation of a range of pore sizes would be both 450 easily achievable as well as beneficial to the field. 
452 Based on the 2D studies that have investigated the impact of geometry on MSC behavior [10, 12,

453 13], we hypothesized that the cubic pore geometry would best support osteogenesis, while the

454 cylindrical pore geometry would promote the adipogenic and chondrogenic groups.

455 Chondrogenic differentiation is typically completed using a 3D high density pellet culture system

456 [38] which promotes increased cell-cell connections to facilitate cell signaling necessary for

457 differentiation. Likewise, adipogenesis is induced using high density culture and has been

458 shown to be promoted in environments with less cytoskeletal stress, such as a rounded geometry

459 [10]. Environments with increased tension, such as a square or cubic geometry have been shown

460 to support osteogenesis, and cytoskeletal tension has been found to be an important factor in the

461 regulation of cell fate [13]. Here in 3D scaffolds, we observe a nearly opposite trend. While the

462 results of histological analysis show no differences between groups, qRT-PCR analysis shows

463 that for both the adipogenic and chondrogenic groups, the cubic pore geometry results in a

464 significant upregulation of the expression of key markers of cell phenotype for each respective

465 lineage. For the osteogenic group, there is an initial upregulation of osteogenic expression in the

466 cylindrical pore group at day 7, with ALP expression, a marker known to peak at day 7 during

467 osteogenesis, significantly upregulated in the cylindrical pore geometry. However, by day 21,

468 the cubic pore scaffold shows a significantly higher number of calcified regions, quantified using

469 alizarin red staining. Overall, the cubic pore geometry appears to have a greater influence over

470 the promotion of differentiation regardless of lineage. We hypothesize that the success of the

471 cubic pore scaffolds may be attributed to the increased elastic modulus as well as the high level

472 of pore size and porosity uniformity that was achieved with this design.

\section{5. Conclusion}

475 In the work presented here, we were able to show the ability to successfully and reproducibly

476 print 3D scaffolds with an interconnected porous network. Optimization of post processing

477 methods provides a clean scaffold with little to no uncrosslinked resin remaining that encourages

478 cell attachment and proliferation. The mechanical properties of the scaffolds can be tuned by

479 varying the length of post curing as well as the molecular weight of the polymer. This would

480 allow for the material properties to be altered based on the application, providing a means to

481 match the mechanical properties of the scaffold with the target tissue. Cubic pore scaffolds were

482 supportive of MSC enrichment from unprocessed human bone marrow, indicating the potential 
483 to utilize 3D printed PPF scaffolds for direct MSC isolation and differentiation. In addition, the 484 3D environments studied here were supportive of human MSC differentiation down all three of 485 the main mesenchymal lineages. Cubic pore geometry was shown to significantly support 486 adipogenic and chondrogenic lineage commitment on the gene expression level compared to its 487 cylindrical pore counterpart, although these differences were not detected at the protein level. 488 For osteogenic differentiation, gene expression data suggested an early role for cylindrical pore 489 geometry, while protein level expression appears to be influenced by cubic pore geometry. The

490 development of a tunable 3D culture system for optimized culture of MSCs would have great 491 potential in the field of tissue engineering and regenerative medicine.

492

\section{6. Acknowledgements}

494 Research reported in this publication was supported by the National Science Foundation/Food 495 and Drug Administration Scholar-in-Residence Program (CBET 1238398). The authors wish to 496 thank Dr. Srinidhi Nagaraja for his technical assistance obtaining and analyzing the results of $497 \mu \mathrm{CT}$ and Dr. Girish Kumar for his technical assistance with the flow cytometry experiments. 498 


\section{References}

1. Prockop, D.J., Marrow stromal cells as stem cells for nonhematopoietic tissues. Science, 1997. 276(5309): p. 71-4.

2. Dominici, M., et al., Minimal criteria for defining multipotent mesenchymal stromal cells. The International Society for Cellular Therapy position statement. Cytotherapy, 2006. 8(4): p. 315-7.

3. Lv, F.J., et al., Concise review: the surface markers and identity of human mesenchymal stem cells. Stem Cells, 2014. 32(6): p. 1408-19.

4. Baksh, D., L. Song, and R.S. Tuan, Adult mesenchymal stem cells: characterization, differentiation, and application in cell and gene therapy. Journal of cellular and molecular medicine, 2004. 8(3): p. 301-16.

5. Bara, J.J., et al., Concise review: Bone marrow-derived mesenchymal stem cells change phenotype following in vitro culture: implications for basic research and the clinic. Stem Cells, 2014. 32(7): p. 1713-23.

6. Santos, F., et al., Toward a clinical-grade expansion of mesenchymal stem cells from human sources: a microcarrier-based culture system under xeno-free conditions. Tissue Eng Part C Methods, 2011. 17(12): p. 1201-10.

7. Goh, T.K., et al., Microcarrier culture for efficient expansion and osteogenic differentiation of human fetal mesenchymal stem cells. Biores Open Access, 2013. 2(2): p. 84-97.

8. Hervy, M., et al., Long term expansion of bone marrow-derived hMSCs on novel synthetic microcarriers in xeno-free, defined conditions. PLoS One, 2014. 9(3): p. e92120.

9. Lane, S.W., D.A. Williams, and F.M. Watt, Modulating the stem cell niche for tissue regeneration. Nat Biotechnol, 2014. 32(8): p. 795-803.

10. Kilian, K.A., et al., Geometric cues for directing the differentiation of mesenchymal stem cells. Proc Natl Acad Sci U S A, 2010. 107(11): p. 4872-7.

11. Engler, A.J., et al., Matrix elasticity directs stem cell lineage specification. Cell, 2006. 126(4): p. 677-89.

12. Lee, J., et al., Directing stem cell fate on hydrogel substrates by controlling cell geometry, matrix mechanics and adhesion ligand composition. Biomaterials, 2013. 34(33): p. 8140-8.

13. McBeath, R., et al., Cell shape, cytoskeletal tension, and RhoA regulate stem cell lineage commitment. Dev Cell, 2004. 6(4): p. 483-95.

14. Zhang, Q., et al., Pore size effect of collagen scaffolds on cartilage regeneration. Acta Biomater, 2014. 10(5): p. 2005-13.

15. Lien, S.M., L.Y. Ko, and T.J. Huang, Effect of pore size on ECM secretion and cell growth in gelatin scaffold for articular cartilage tissue engineering. Acta Biomater, 2009. 5(2): p. 670-9.

16. Woodfield, T.B., et al., Design of porous scaffolds for cartilage tissue engineering using a three-dimensional fiber-deposition technique. Biomaterials, 2004. 25(18): p. 4149-61.

17. Wang, M.O., et al., Evaluating Changes in Structure and Cytotoxicity During In Vitro Degradation of Three-Dimensional Printed Scaffolds. Tissue engineering. Part A, 2015.

18. Wang, M.O., et al., Evaluating 3D-printed biomaterials as scaffolds for vascularized bone tissue engineering. Adv Mater, 2015. 27(1): p. 138-44. 
19. Kim, K., et al., The influence of stereolithographic scaffold architecture and composition on osteogenic signal expression with rat bone marrow stromal cells. Biomaterials, 2011. 32(15): p. 3750-63.

20. Duarte Campos, D.F., et al., The stiffness and structure of three-dimensional printed hydrogels direct the differentiation of mesenchymal stromal cells toward adipogenic and osteogenic lineages. Tissue engineering. Part A, 2015. 21(3-4): p. 740-56.

21. Castro, N.J., R. Patel, and L.G. Zhang, Design of a Novel 3D Printed Bioactive Nanocomposite Scaffold for Improved Osteochondral Regeneration. Cellular and molecular bioengineering, 2015. 8(3): p. 416-432.

22. Zhang, W., et al., The effect of interface microstructure on interfacial shear strength for osteochondral scaffolds based on biomimetic design and $3 D$ printing. Materials science \& engineering. C, Materials for biological applications, 2015. 46: p. 10-5.

23. Fisher, J.P., D. Dean, and A.G. Mikos, Photocrosslinking characteristics and mechanical properties of diethyl fumarate/poly(propylene fumarate) biomaterials. Biomaterials, 2002. 23(22): p. 4333-43.

24. Fisher, J.P., et al., Soft and hard tissue response to photocrosslinked poly(propylene fumarate) scaffolds in a rabbit model. J Biomed Mater Res, 2002. 59(3): p. 547-56.

25. Wang, M.O., et al., Evaluation of the in vitro cytotoxicity of cross-linked biomaterials. Biomacromolecules, 2013. 14(5): p. 1321-9.

26. Kasper, F.K., et al., Synthesis of poly(propylene fumarate). Nat Protoc, 2009. 4(4): p. 518-25.

27. Bracaglia, L.G., et al., Reinforced pericardium as a hybrid material for cardiovascular applications. Tissue Eng Part A, 2014. 20(21-22): p. 2807-16.

28. Yeatts, A.B. and J.P. Fisher, Tubular perfusion system for the long-term dynamic culture of human mesenchymal stem cells. Tissue Eng Part C Methods, 2011. 17(3): p. 337-48.

29. Ferlin, K.M., et al., Development of a dynamic stem cell culture platform for mesenchymal stem cell adhesion and evaluation. Mol Pharm, 2014. 11(7): p. 2172-81.

30. Dean, D., et al., Continuous Digital Light Processing (cDLP): Highly Accurate Additive Manufacturing of Tissue Engineered Bone Scaffolds. Virtual and physical prototyping, 2012. 7(1): p. 13-24.

31. Mareschi, K., et al., Multipotent mesenchymal stromal stem cell expansion by plating whole bone marrow at a low cellular density: a more advantageous method for clinical use. Stem Cells Int, 2012. 2012: p. 920581.

32. Papadimitropoulos, A., et al., Expansion of human mesenchymal stromal cells from fresh bone marrow in a 3D scaffold-based system under direct perfusion. PLoS One, 2014. 9(7): p. e102359.

33. Lipowiecki, M., et al., Permeability of rapid prototyped artificial bone scaffold structures. J Biomed Mater Res A, 2014. 102(11): p. 4127-35.

34. Phipps, M.C., et al., Increasing the pore sizes of bone-mimetic electrospun scaffolds comprised of polycaprolactone, collagen I and hydroxyapatite to enhance cell infiltration. Biomaterials, 2012. 33(2): p. 524-34.

35. Huri, P.Y., et al., Scaffold pore size modulates in vitro osteogenesis of human adiposederived stem/stromal cells. Biomed Mater, 2014. 9(4): p. 045003.

36. Jeong, C.G. and S.J. Hollister, Mechanical and biochemical assessments of threedimensional poly(1,8-octanediol-co-citrate) scaffold pore shape and permeability effects 
on in vitro chondrogenesis using primary chondrocytes. Tissue Eng Part A, 2010. 16(12): p. 3759-68.

37. Jeong, C.G., H. Zhang, and S.J. Hollister, Three-dimensional poly(1,8-octanediol-cocitrate) scaffold pore shape and permeability effects on sub-cutaneous in vivo chondrogenesis using primary chondrocytes. Acta Biomater, 2011. 7(2): p. 505-14.

38. Yang, Z., J.F. Schmitt, and E.H. Lee, Immunohistochemical analysis of human mesenchymal stem cells differentiating into chondrogenic, osteogenic, and adipogenic lineages. Methods Mol Biol, 2011. 698: p. 353-66. 


\section{Tables}

Table 1: Flow Cytometry Analysis for the Panel of Positive Surface Markers For Human MSCs Following Bone Marrow Seeding on Unmodified and Biomolecule Modified 3D

Printed PPF Scaffolds. Data is shown as the percent of P1, which was the cell gate set using human MSC positive controls. On day 1, both modified and unmodified scaffolds demonstrate expression levels equivalent to the TCPS control. Surface modified scaffolds have a marginally increased expression of positive surface markers. Scaffolds modified with a mixture of collagen and HA, as well as scaffolds modified to contain the growth factor TGF- $\beta 3$ showed the highest level of expression at day 1 , with levels above that of the control. On day 7, all experimental groups show expression levels similar to the control. Both the unmodified scaffold group and the collagen modified scaffold group exhibit expression levels that are increased over the control, with the collagen modified scaffold group having the highest overall expression compared to all groups.

\begin{tabular}{|c|c|c|c|}
\hline Percent of P1 (cell gate) & CD90 & CD73 & CD105 \\
\hline On day 1: & 8.6 & 61.4 & 58.2 \\
\hline Unmodified scaffold & 19.8 & 49.9 & 54.5 \\
\hline Collagen modified scaffold & 32.3 & 63.9 & 68.1 \\
\hline Collagen + HA modified scaffold & 24.5 & 66.0 & 66.1 \\
\hline TGF- $\beta 3$ modified scaffold & 15.7 & 47.8 & 52.3 \\
\hline TCPS & 17.5 & 61.2 & 71.7 \\
\hline On day 7: & 35.0 & 67.7 & 75.3 \\
\hline Unmodified scaffold & 18.7 & 57.3 & 54.6 \\
\hline Collagen modified scaffold & 21.2 & 59.5 & 72.5 \\
\hline Collagen + HA modified scaffold & 25.2 & 58.6 & 60.2 \\
\hline TGF- $\beta 3$ modified scaffold & & & \\
\hline TCPS & & & \\
\hline
\end{tabular}




\section{Figure Captions}

Figure 1: 3D Printed Scaffolds. (A) Analysis of the final designs of each pore geometry including 3D images resulting from $\mu \mathrm{CT}$ imaging. The results from $\mu \mathrm{CT}$ show structures with highly defined and interconnecting pores. (B) The tabulated pore size and porosity are close to the theoretical for both pore geometries.

Figure 2: Post Printing Processing and Characterization of Scaffolds. (A) Modulus resulting from compressive testing of scaffolds exposed to an increasing total energy delivered through post processing UV crosslinking. There were no statistical differences between 25 and $100 \%$ acetone washes, however, as the total energy increased there was a significant increase in the modulus of the scaffold up to $1100 \mathrm{~J}$ ( $\mathrm{p}<0.05$ ). Between 1100 and $2200 \mathrm{~J}$, there were no significant differences ('*', $\mathrm{p}>0.05$ ). The PPF used in this experiment had a MW=1133 Da. (B) Modulus of cubic and cylindrical pore scaffolds subjected to a wash of $30 \mathrm{~min}$ IPA, $30 \mathrm{~min}$ $100 \%$ acetone, $110 \mathrm{~J}$, and $24 \mathrm{~h} 70 \%$ ethanol. The MW of PPF for this experiment was $1632 \mathrm{Da}$. Cubic pore scaffolds exhibited a statistically greater elastic modulus when compared to the cylindrical pore scaffolds ( ' $*,, p<0.05)$. The sol fraction of all scaffolds was less than $5 \%$.

Figure 3: Cell Viability on 3D Printed PPF Scaffolds. (A) XTT data following 1, 4, and 7 days of culture. ' $*$ ' indicates samples on day 7 of both groups were statistically greater than day 1 ( $\mathrm{p}<0.05)$. There were no statistical differences between cells grown on 3D printed scaffolds and the control at any timepoint. (B) Live-dead viability staining of samples at days 1, 4, and 7 of culture. The cells exhibit high viability (green) and localization within the pores of the scaffolds. Scale bar $=100 \mu \mathrm{m}$.

Figure 4: qRT-PCR Assessment of MSC Differentiation on 3D Printed Scaffolds. (A and B) Fold change in expression of osteogenic markers alkaline phosphatase (ALP) and osteopontin (OPN). For both markers, the cylindrical pore group at day 7 exhibited a statistically greater expression level compared to all other groups. (C) Fold change in the expression of the adipogenic marker, adiponectin. At day 21, the cubic pore group showed the highest expression. (D) Fold change in the expression of type II collagen, a marker for chondrogenesis. At day 21, the cubic pore group had statistically greater expression compared to the cylindrical pore group. '*' indicates $\mathrm{p}<0.05$.

Figure 5: Quantification of Histological Assessment of MSC Differentiation on 3D Printed Scaffolds. (A) Comparison of the number of calcified regions, an indicator of osteogenesis. Data is shown as the number of calcified regions per $\mathrm{mm}^{2}$. At day 21, the cubic pore geometry had a statistically greater ('*' indicates $\mathrm{p}<0.05)$ number of calcified regions compared to the cylindrical pore group. (B) For adipogenic samples, the percentage of oil red o positive cells are reported. There was no statistical difference between pore geometries at day $21(\mathrm{p}>0.05)$. (C) Quantification of chondrogenesis was computed by dividing the number of proteoglycan positive regions by the number of cells. At day 21 there was no statistical difference between pore geometries $(\mathrm{p}>0.05)$. 


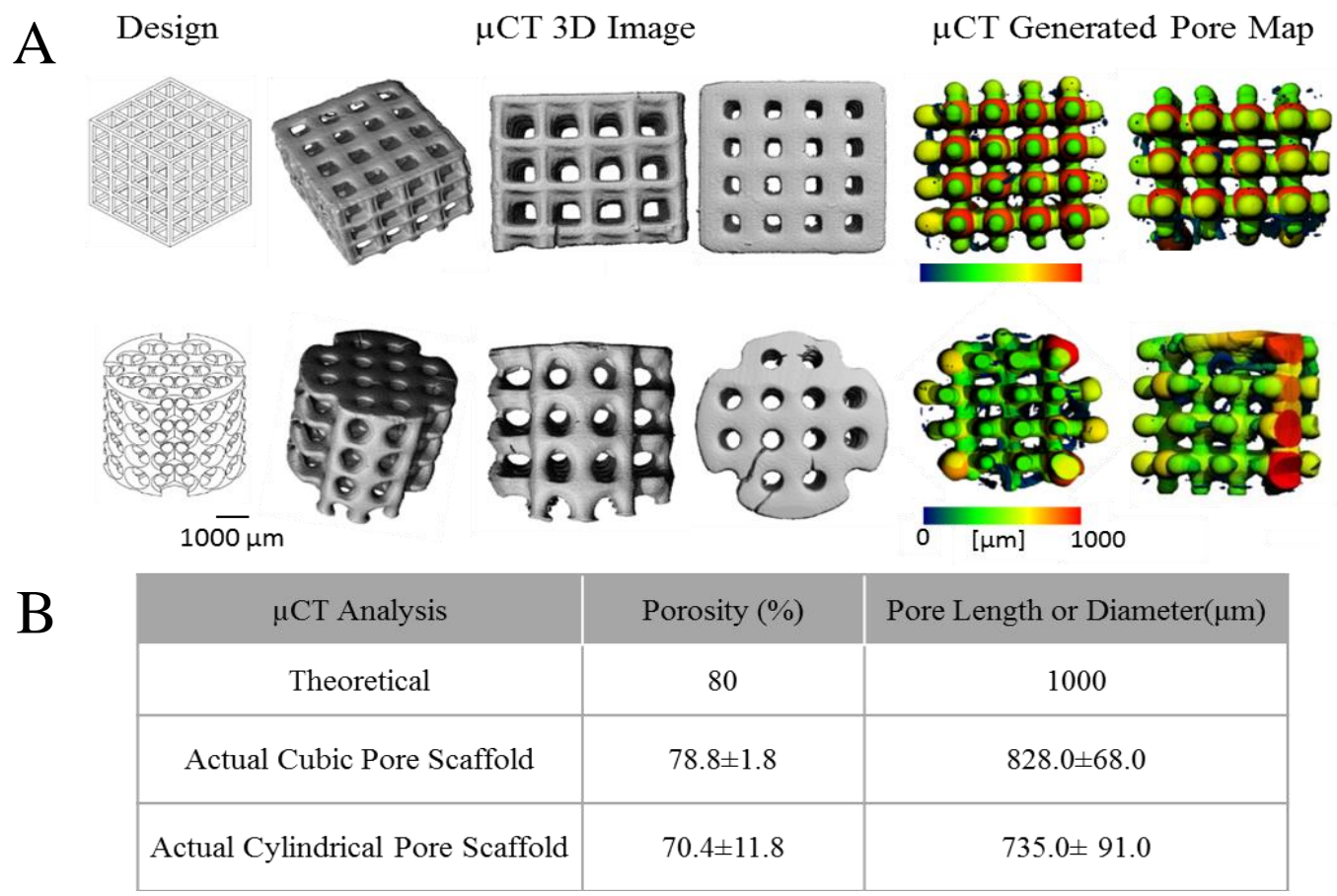

Figure 1 
A

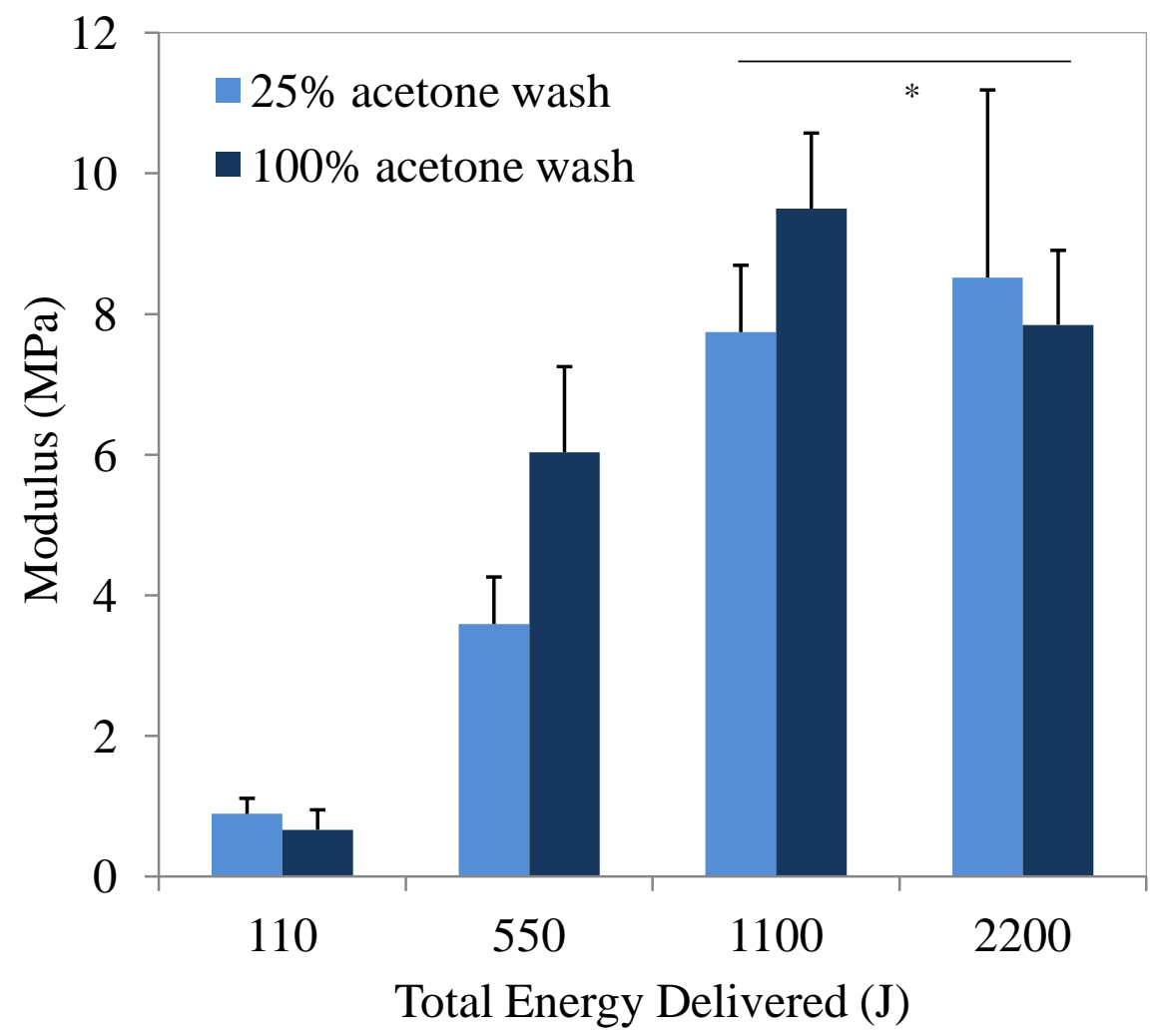

B

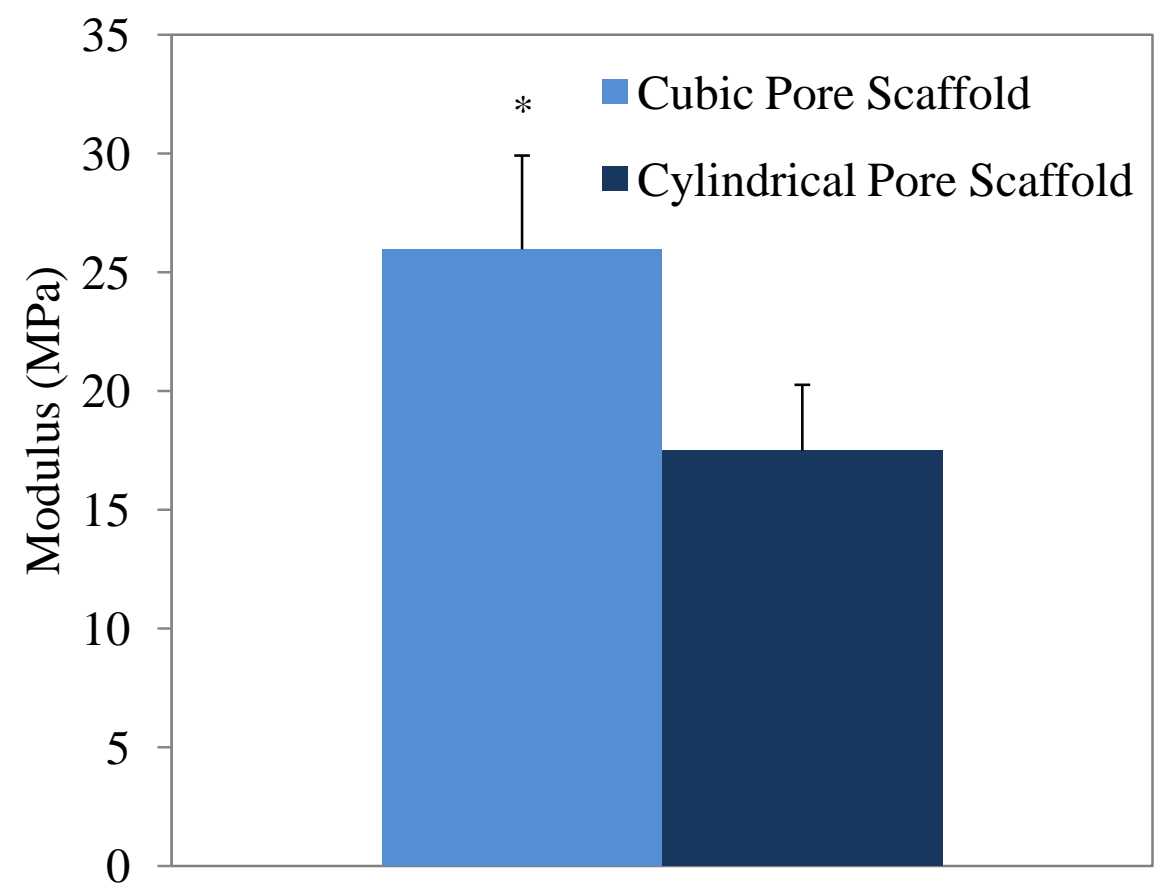

Figure 2 
A

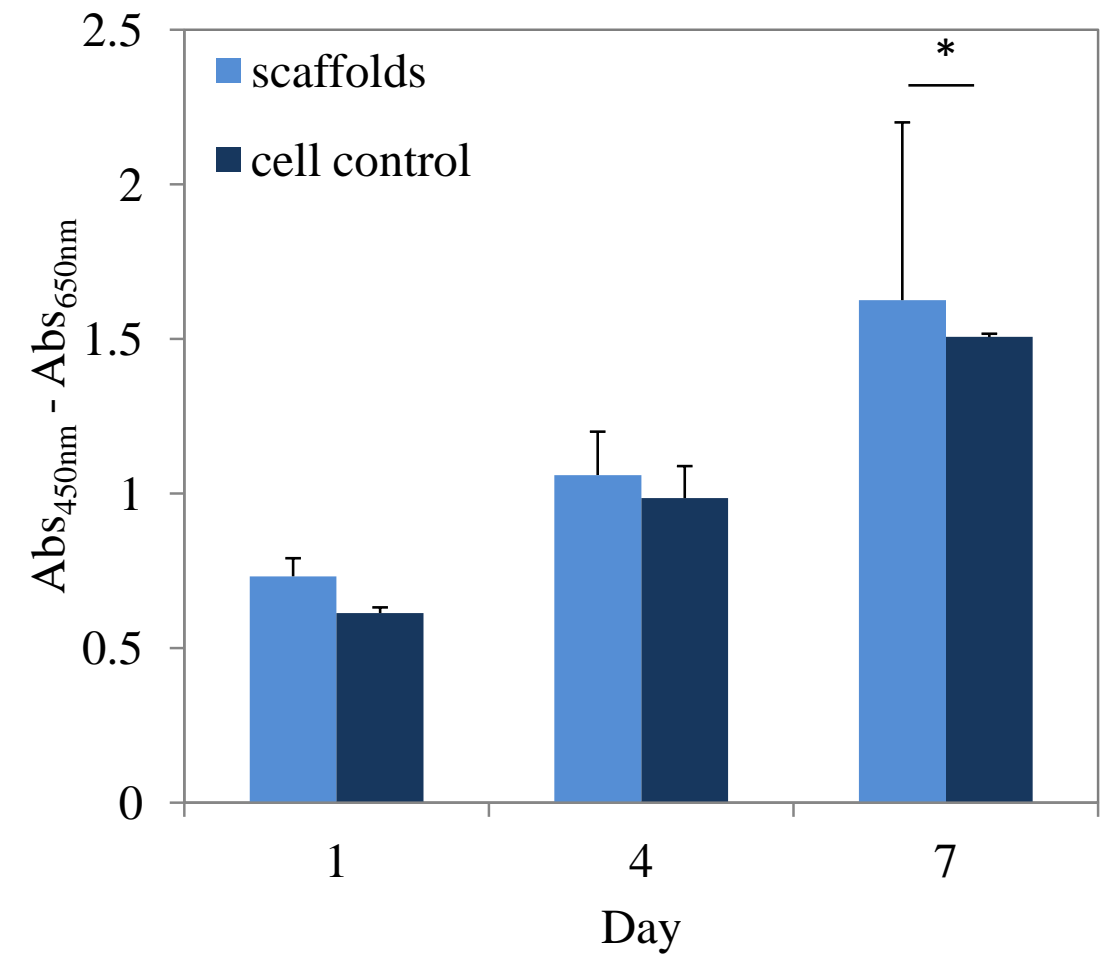

B

Figure 3

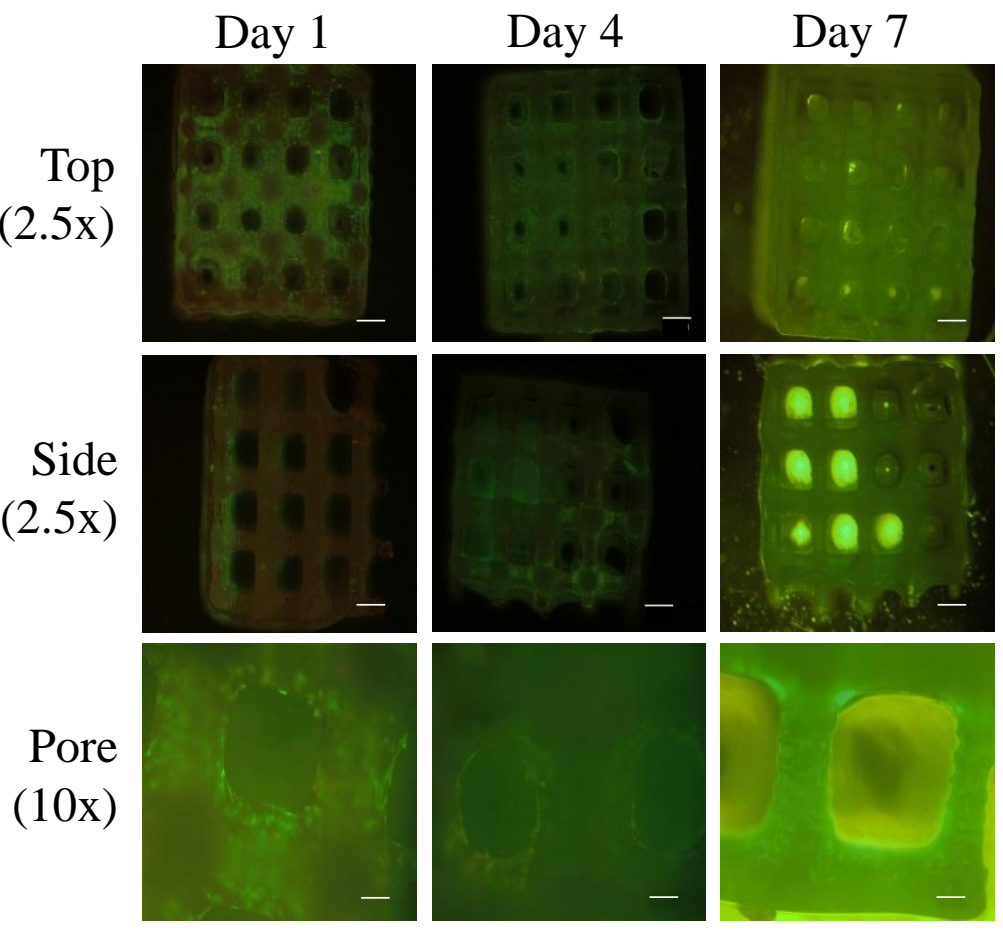



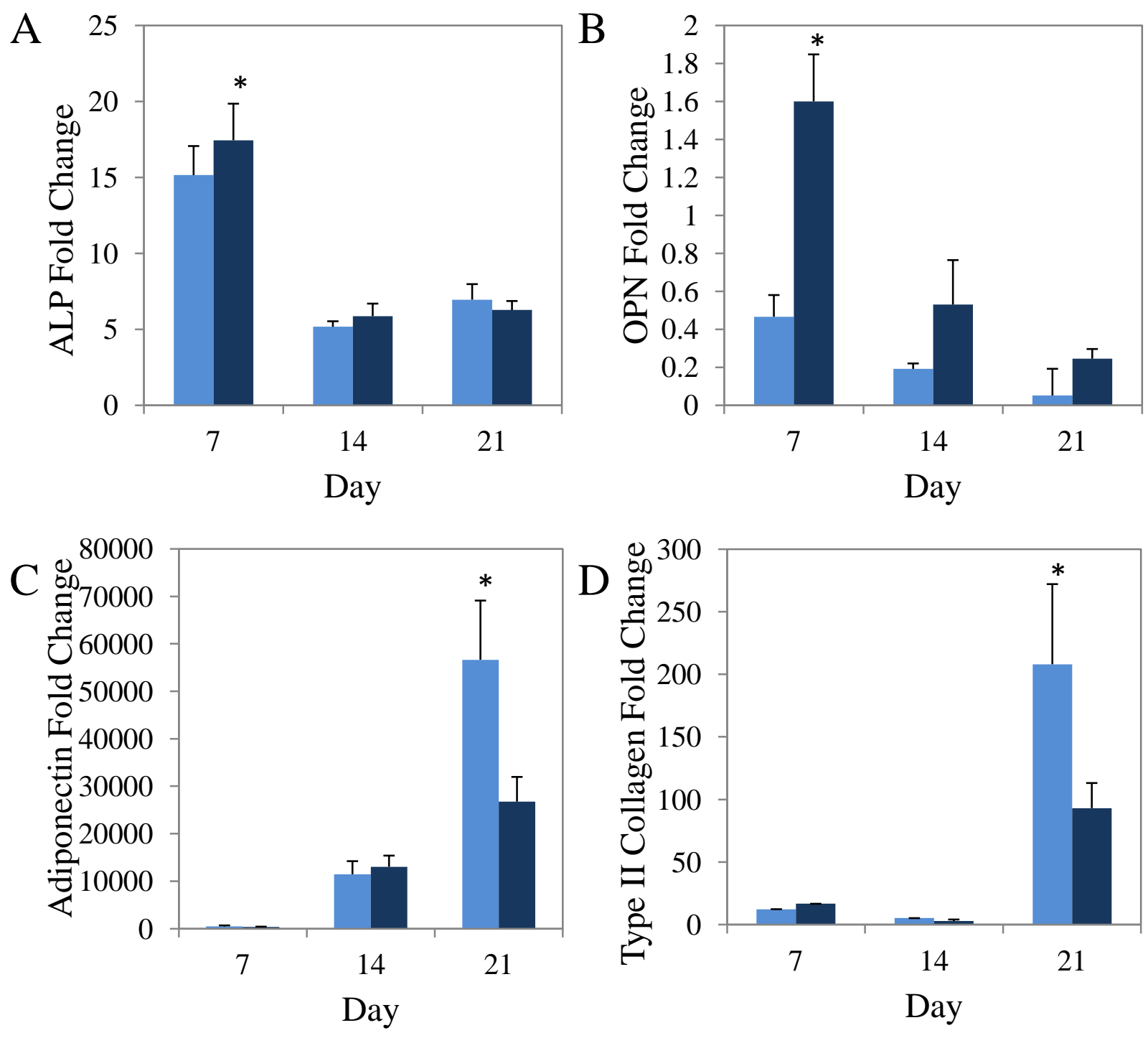

• Cubic Pore Scaffold —Cylindrical Pore Scaffold

Figure 4 
A

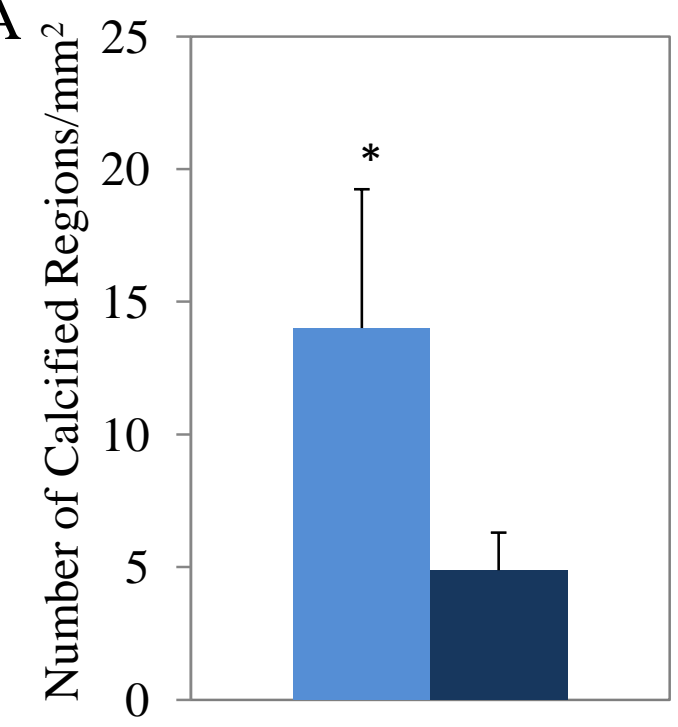

C

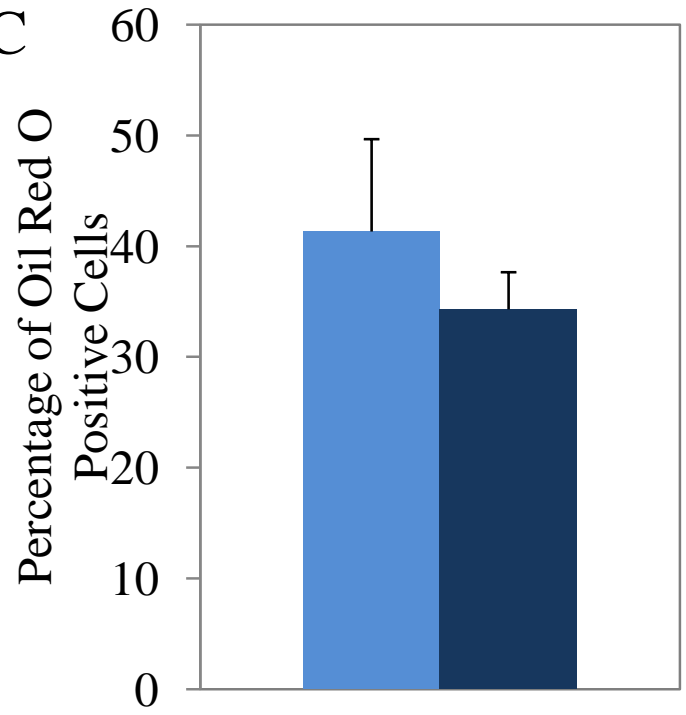

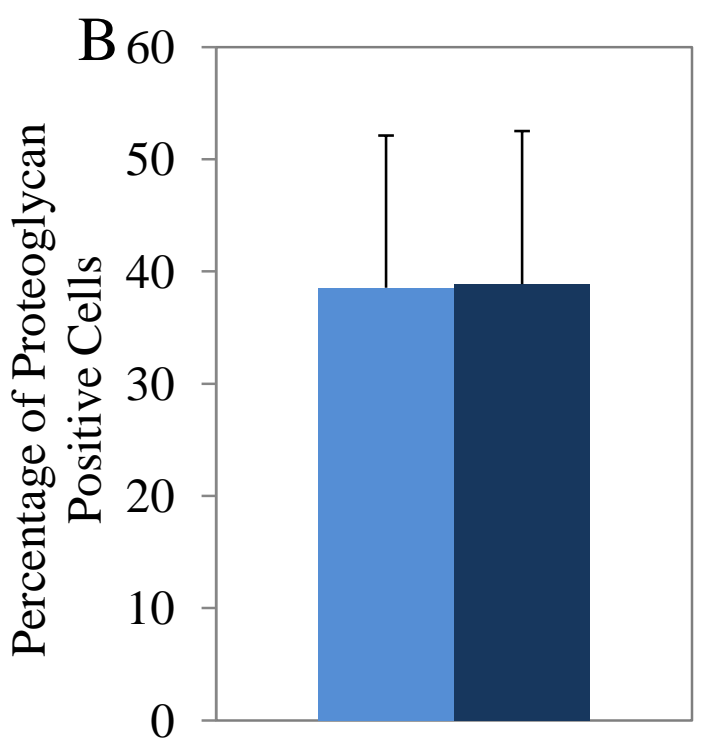

- Cubic Pore Scaffold

- Cylindrical Pore Scaffold

Figure 5 
Graphical Abstract

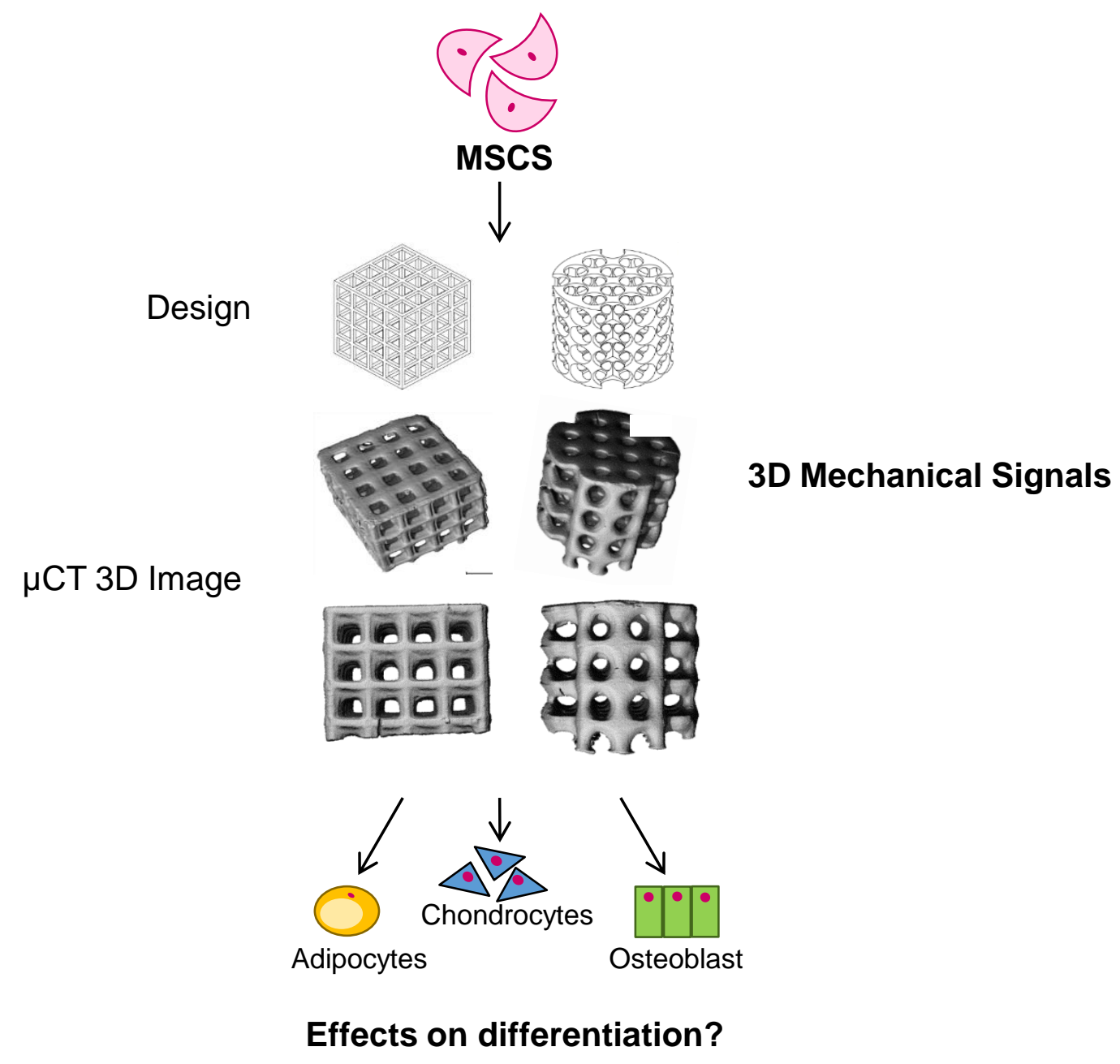

